\title{
Progressive left-sided facial swelling and proptosis
}

\author{
Ritu Kumar $\mathrm{MD}^{1}$, Naheed A Lakhani MD MPH${ }^{2}$, Umesh Narsinghani $\mathrm{MD}^{3}$
}

\section{CASE PRESENTATION}

A previously healthy 87 -year-old African-American man presented to an urgent care facility with left nasal congestion. He reported a twoweek history of nosebleeds, low-grade fever, night sweats and general malaise. He was treated with intravenous ceftriaxone and prescribed oral levofloxacin for suspected acute bacterial sinusitis. Due to worsening symptoms, left eye swelling and continued bloody nasal discharge, he went to a local emergency department and was admitted to the hospital and treated with intravenous ampicillin plus sulbactam for community-acquired acute bacterial rhinosinusitis; however, despite empirical therapy, his facial swelling progressed to involve the periorbital region. He developed left facial numbness, proptosis and decreased vision in the left eye (Figure 1).

On examination, he was afebrile and his heart rate was 82 beats/min, respiratory rate was 23 breaths/min and blood pressure was 130/86 mmHg. The patient was alert and cooperative. He had a small amount of bloody nasal discharge and left-sided facial swelling with tenderness noted in the maxillary area. Additional findings included left eye swelling without erythema that limited eye opening and examination. There was no pharyngeal erythema or oral lesions. He had no cervical adenopathy or organomegaly. There were no cutaneous or musculoskeletal findings. His respiratory, cardiovascular, abdominal and neurological examination were unremarkable. He had a white blood cell count of $170 \times 10^{9} / \mathrm{L}$ with $60 \%$ neutrophils, $8 \%$ bands, $16 \%$ monocytes, $14 \%$ metamyelocytes and $2 \%$ lymphocytes. He was anemic and thrombocytopenic; his hemoglobin level was $74 \mathrm{~g} / \mathrm{L}$ and platelet count was $13 \times 10^{9} / \mathrm{L}$. His prothrombin time was $17.9 \mathrm{~s}$, partial thromboplastin time $34.5 \mathrm{~s}$ and international normalized ratio was 1.44 . His plasma chemistry, including liver function tests and blood glucose measurements, were normal. Magnetic resonance imaging of the brain with contrast demonstrated the presence of extensive left-sided sinusitis involving the frontal, sphenoid, ethmoid and maxillary sinuses, with intraorbital extension of infection to the left medial superior orbit.

As part of management, debridement and left inferior turbinate biopsy was performed by ear, nose and throat surgery.

Based on the patient's history, physical examination and clinical findings, what is the most likely diagnosis?

\section{DIAGNOSIS}

The patient was diagnosed with rhino-orbital-cerebral mucormycosis with acute myeloid leukemia. Left inferior turbinate biopsy revealed scattered ribbon-like fungal microorganisms demonstrating $90^{\circ}$ branching without septae in a background of necrosis consistent with mucormycosis (Figure 2). Peripheral blood flow cytometry was consistent with acute myeloid leukemia.

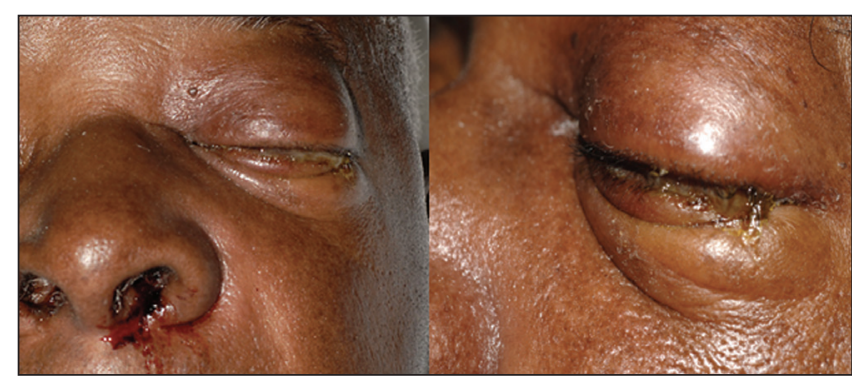

Figure 1) Left facial and periorbital swelling

\section{DISCUSSION}

Fungal rhinosinusitis encompasses a wide variety of fungal infections that range from merely irritating to a rapidly progressive and often fatal course. Fungal colonization of the upper and lower airways is a common occurrence because fungal spores are constantly inhaled into the sinuses and lungs (1).

Mucormycosis is an opportunistic infection caused by fungi of the order Mucorales, and has angioinvasive and proliferative pathology in the susceptible host. Mucorales are ubiquitous in nature, and can be found on decaying vegetation and in the soil. The incidence of mucormycosis in adult leukemia patients is approximately $2 \%(1)$, and patients with hematological malignancies, especially those who develop graft-versus-host disease due to hematopoietic cell transplant, are highly susceptible (2). Typically, mucormycosis occurs in patients with diabetes mellitus (especially those who present with ketoacidosis), patients on glucocorticoid therapy, transplant recipients, patients with iron overload receiving deferoxamine therapy and AIDS patients (3). The most common clinical presentation of mucormycosis is rhinoorbital-cerebral infection, which is presumed to start with inhalation of spores into the paranasal sinuses of a susceptible host (4). Mucormycosis is characterized by infarction and necrosis of host tissues resulting from invasion of the vasculature by the spores. Infection usually presents as nasal congestion, sinusitis with fever, purulent nasal discharge and headaches. It then spreads to contiguous structures, such as the palate, orbit and brain, and usually progresses rapidly. Signs of orbital involvement include periorbital edema, proptosis, blindness and ophthalmoplegia (5). The nares and oral cavity should be carefully examined for necrotic areas. The diagnosis of rhino-orbitalcerebral mucormycosis is based on the identification of organisms in tissue by histopathology with culture confirmation; thus, early nasal endoscopic evaluation by an otolaryngologist is critical. Mortality rates in patients with hematological malignancies and mucormycosis

${ }^{1}$ Department of Internal Medicine, Mercer University School of Medicine, Macon; ${ }^{2}$ Department of Family Medicine, Emory University School of Medicine, Dunwoody; ${ }^{3}$ Department of Pediatrics, Mercer University School of Medicine, Macon, Georgia, USA

Correspondence: Dr Ritu Kumar, Mercer University School of Medicine, Department of Internal Medicine, Infectious Disease, 707 Pine Street, Macon, Georgia 31201 USA. Telephone 478-301-5800, fax 478-301-5841, e-mail kumar_ra@mercer.edu 


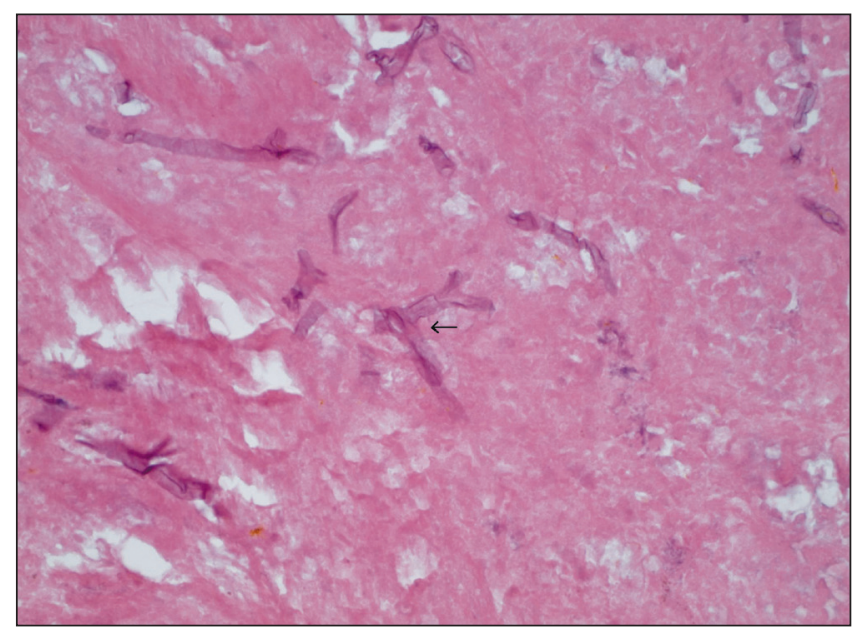

Figure 2) Scattered ribbon-like aseptate fungal hyphae demonstrating $90^{\circ}$ branching in a background of necrosis

have been reported to be as high as $80 \%(6,7)$. Surgical intervention and debridement along with antifungal therapy using intravenous amphotericin B is the gold standard for eradicating the infection $(8,9)$. Posaconazole can be used as a step-down therapy in patients who have responded to liposomal amphotericin B. In addition, treatment of predisposing factors, such as hyperglycemia, metabolic acidosis and neutropenia, is essential. In patients with rhino-orbital-cerebral infections, the most significant factors associated with death were delayed diagnosis, central nervous system involvement, leukemia, renal disease

\section{REFERENCES}

1. Kurosawa M, Yonezumi M, Hashino S, et al. Epidemiology and treatment outcome of invasive fungal infections in patients with hematological malignancies. Int J Hematol 2012;96:748-57.

2. Lanternier F, Sun HY, Ribaud P, Singh N, Kontoyiannis DP, Lortholary O. Mucormycosis in organ and stem cell transplant recipients. Clin Infect Dis 2012;54:1629.

3. Shinde RV, Karande GS, Mohite ST, Patil SR. Rhino-orbital mucormycosis in diabetes mellitus. J Clin Diagn Res 2013;7:1145-7.

4. Anane S, Kaouech E, Belhadj S, et al. Rhino-orbito-cerebral mucormycosis in the diabetic: A better known pathology in Tunisia. Ann Biol Clin (Paris) 2009;67:325-32.

5. Petrikkos G, Skiada A, Lortholary O, et al. Epidemiology and clinical manifestations of mucormycosis. Clin Infect Dis 2012;54(Suppl 1):S23. and treatment with deferoxamine. Our patient opted for palliative measures $(9,10)$.

Common and selected differential diagnosis includes:

- Acute bacterial rhinosinusitis. Acute bacterial rhinosinusitis occurs most commonly as a complication of viral infection such as the common cold or allergic rhinitis. Symptoms typically improve with supportive measures and antibiotic treatment.

- Basal cell carcinoma. This is a common malignancy typically observed in Caucasians; it appears on the surface of the skin, and is rarely invasive.

- Chronic and compulsive intranasal use of cocaine. This condition causes progressive damage of the mucosa and perichondrium, leading to ischemic necrosis of septal cartilage and perforation of the nasal septum.

- Leukemoid reaction or hyperleukocytosis. In patients with hyperleukocytosis, white blood cell count is $>100 \times 10^{9} / \mathrm{L}$ and lifethreatening pulmonary and central nervous system disease results due to vascular occlusion of the leukocytes.

\section{CONCLUSION}

Mucormycosis should be suspected in high-risk patients, especially those who have diabetes mellitus with ketoacidosis, and immunocompromised hosts who present with sinusitis and necrotic tissue in the nose or palate. Timely endoscopic evaluation of the sinuses should be performed to identify tissue destruction and to obtain specimens for histopathology. The diagnosis of mucormycosis depends on identification of organisms and culture confirmation. Treatment of mucormycosis involves a combination of surgical debridement of the involved tissues and antifungal therapy. Despite diagnosis and aggressive combined surgical and medical therapy, the prognosis for recovery from mucormycosis is universally poor.

6. Santos Gorjón P, Blanco Pérez P, Batuecas Caletrío A, Muñoz Herrera AM, Sánchez González F, de la Fuente Cañibano R. Rhino-orbito-cerebral mucormycosis, a retrospective study of 7 cases. Acta Otorrinolaringol Esp 2010;61:48-53.

7. Xia ZK, Wang WL, Yang RY. Slowly progressive cutaneous, rhinofacial, and pulmonary mucormycosis caused by Mucor irregularis in an immunocompetent woman. Clin Infect Dis 2013;56:993.

8. Weprin BE, Hall WA, Goodman J, Adams GL Long-term survival in rhinocerebral mucormycosis. Case report. J Neurosurg 1998;88:570.

9. Kontoyiannis DP, Lewis RE. How I treat mucormycosis. Blood 2011;118:1216.

10. Roden MM, Zaoutis TE, Buchanan WL, et al. Epidemiology and outcome of zygomycosis a review of 929 reported cases. Clin Infect Dis 2005;41:634-53. 


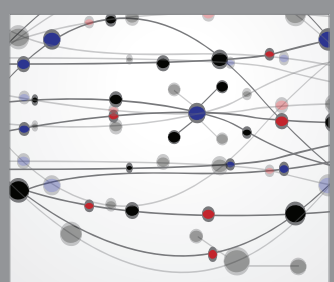

The Scientific World Journal
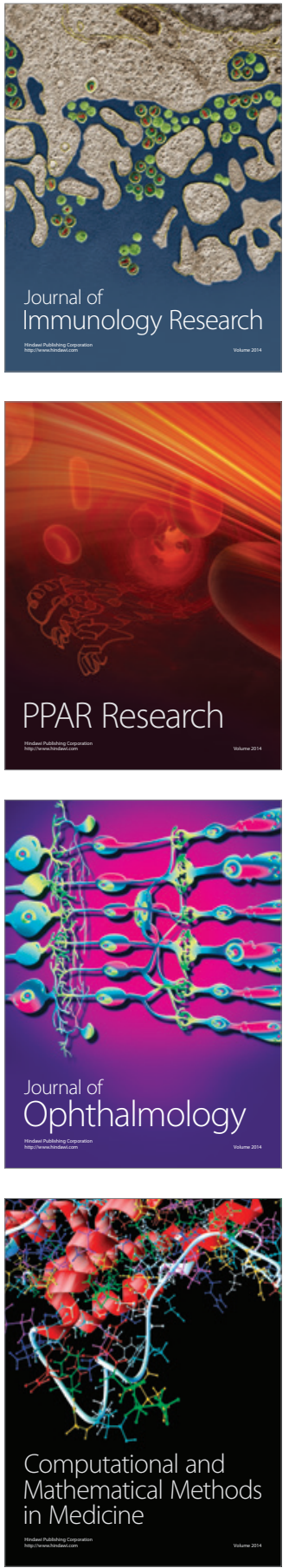

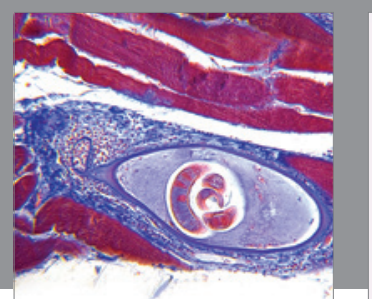

Gastroenterology Research and Practice

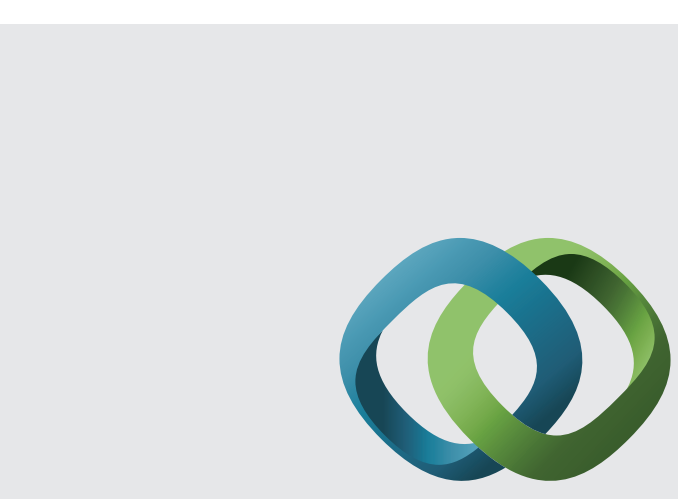

\section{Hindawi}

Submit your manuscripts at

http://www.hindawi.com
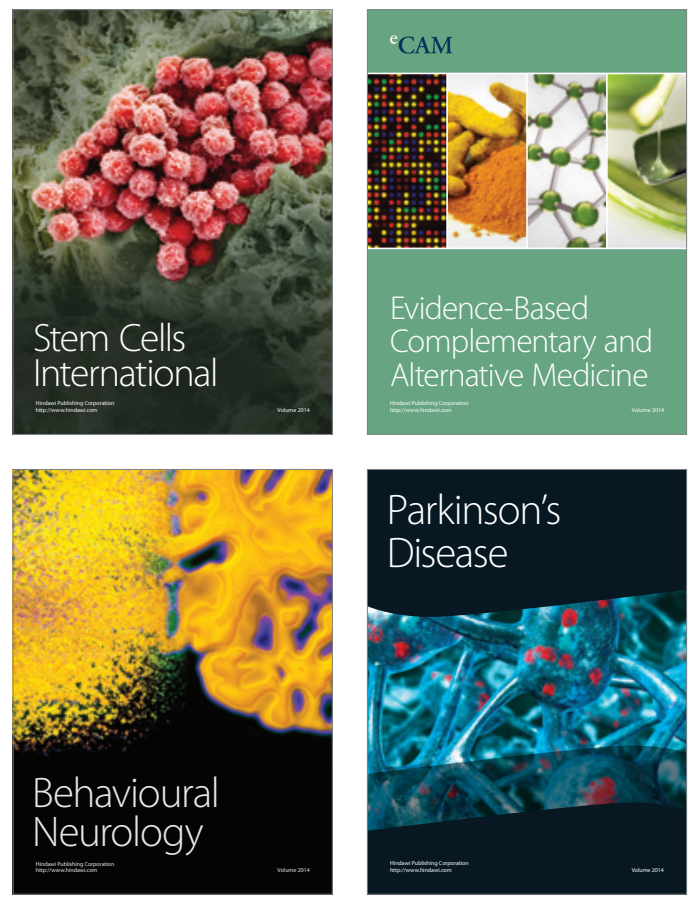
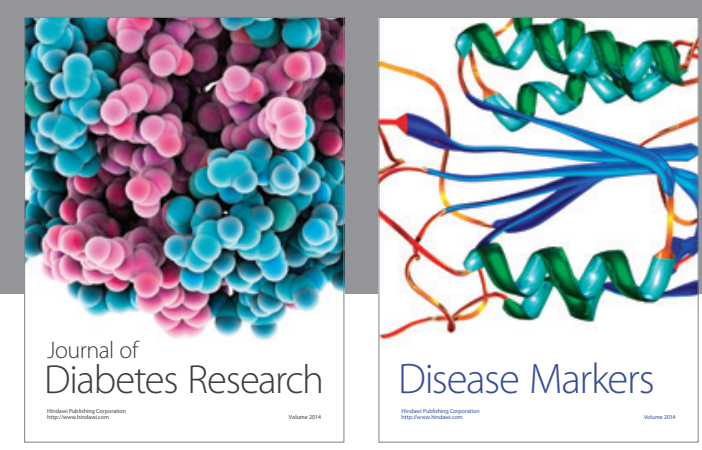

Disease Markers
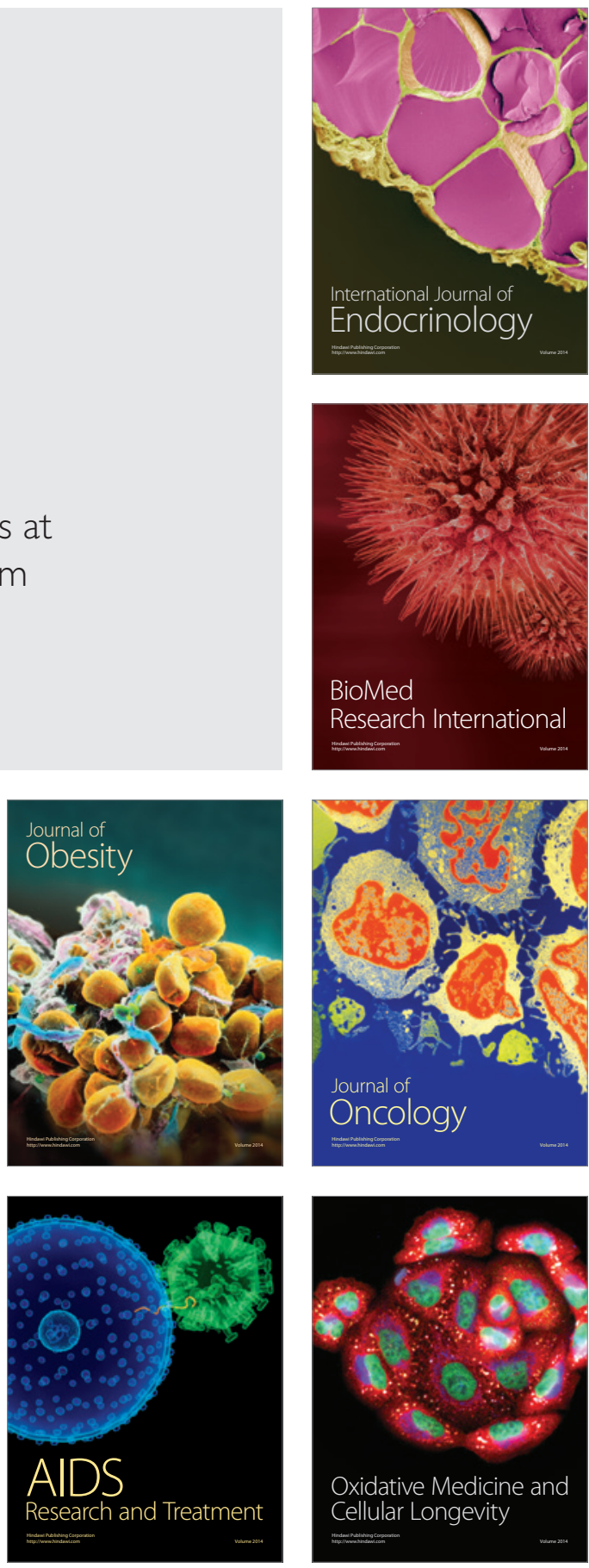